\title{
Qualitative properties of solutions for nonlinear Schrödinger equations with nonlinear boundary conditions on the half-line
}

\author{
Varga K. Kalantarov ${ }^{1,2}$ and Türker Özsari ${ }^{3, a)}$ \\ ${ }^{1}$ Department of Mathematics, Koç University, istanbul, Turkey \\ ${ }^{2}$ Institute of Mathematics and Mechanics, Academy of Sciences of Azerbaijan, \\ Baku, Azerbaijan \\ ${ }^{3}$ Department of Mathematics, İzmir Institute of Technology, İzmir, Turkey
}

(Received 5 August 2015; accepted 26 January 2016; published online 9 February 2016)

\begin{abstract}
In this paper, we study the interaction between a nonlinear focusing Robin type boundary source, a nonlinear defocusing interior source, and a weak damping term for nonlinear Schrödinger equations posed on the infinite half-line. We construct solutions with negative initial energy satisfying a certain set of conditions which blow-up in finite time in the $H^{1}$-sense. We obtain a sufficient condition relating the powers of nonlinearities present in the model which allows construction of blow-up solutions. In addition to the blow-up property, we also discuss the stabilization property and the critical exponent for this model. (C) 2016 AIP Publishing LLC. [http://dx.doi.org/10.1063/1.4941459]
\end{abstract}

\section{INTRODUCTION}

In this paper, we consider the following nonlinear Schrödinger equation (NLS) model posed on the infinite half-line:

$$
\begin{cases}i \partial_{t} u-u_{x x}+k|u|^{p} u+i a u=0, & t>0, x \in I=(0, \infty), \\ u(x, 0)=u_{0}(x), & x>0, \\ u_{x}(0, t)=-\lambda|u(0, t)|^{r} u(0, t), & t>0,\end{cases}
$$

where $u=u(x, t)$ is a complex valued function, the real variables $x$ and $t$ are space and time coordinates, subscripts denote partial derivatives, and $u_{0}$ is the initial state. The constant parameters satisfy $\lambda, p, k, r>0$ and $a \geq 0$. When $\lambda=0$, the boundary condition reduces to the classical homogeneous Neumann boundary condition. When $r=0$, the boundary condition is the classical homogeneous Robin boundary condition. When $\lambda$ and $r$ are both non-zero as in the present case, the boundary condition can be considered a nonlinear variation of the Robin boundary condition.

NLS is a classical field equation whose popularity increased especially when it was shown to be integrable in Ref. 23. Although it has many applications in physics, NLS does not model the evolution of a quantum state, unlike the linear Schrödinger equation. Applications of NLS include transmission of light in nonlinear optical fibers and planar waveguides, small-amplitude gravity waves on the surface of deep inviscid water, and Langmuir waves in hot plasmas. ${ }^{20,13}$ NLS also appears as a universal equation governing the evolution of slowly varying packets of quasi-monochromatic waves in weakly nonlinear dispersive media. ${ }^{20,13}$ Some other interesting applications of NLS include Bose-Einstein condensates, ${ }^{18}$ Davydov's alpha-helix solitons, ${ }^{2}$ and plane-diffracted wave beams in the focusing regions of the ionosphere. ${ }^{10}$

There is a large literature on the qualitative behavior of solutions for NLS. Our particular attention in this paper will be the blow-up and stabilization of solutions at the energy level. The

a) Author to whom correspondence should be addressed. Electronic mail: turkerozsari@iyte.edu.tr 
blow-up theory for nonlinear Schrödinger equations in the presence of a damping term has attracted the attention of several scientists. Some of the major work in this subject are Refs. 22, 8, and 12. Stabilization of solutions for weakly damped nonlinear Schrödinger equations has been studied well with homogeneous boundary conditions (see, for example, Ref. 21). Regarding nonhomogeneous boundary conditions; see Refs. 15-17. The stabilization problem for nonlinear Schrödinger equations has also been studied with locally supported damping (i.e., damping is only effective on a subregion of the given domain), see, for example, Refs. 5-7.

Model (1) with linear main equation $(k=0)$ and no damping $(a=0)$ has been studied in Ref. 1. Local existence and uniqueness of $H^{1}$ solutions have been obtained for sufficiently smooth data $\left(u_{0} \in H^{3}\left(\mathbb{R}_{+}\right)\right)$. For those local solutions, global existence of $H^{1}$ solutions has been obtained for $r<2$ in the case of arbitrarily large data, and for $r=2$ in the case of small data. It has also been shown that solutions with strictly negative energy blow up if $r \geq 2$. We define the energy function associated with (1) by

$$
E(t) \equiv\left\|u_{x}(t)\right\|_{L^{2}(I)}^{2}-\frac{2 \lambda}{r+2}|u(0, t)|^{r+2}+\frac{2 k}{p+2}\|u(t)\|_{L^{p+2}(I)}^{p+2}
$$

for $t \geq 0$. Therefore, $r=2$ was considered to be the critical exponent for the blow-up problem in the linear model. There is another study (see Ref. 11) where the linear Schrödinger equation was considered with nonlinear boundary conditions. In Ref. 11, the authors obtain well-posedness and decay rate estimates at the $L^{2}$-level for the Schrödinger equation with nonlinear, attractive, and dissipative boundary conditions of type $\frac{\partial u}{\partial v}=i g(u)$, where $g$ satisfies some monotonicity conditions. Most recently, the nonlinear Schrödinger equation of cubic type was studied with nonlinear dynamical boundary conditions, which are equivalent to so called (nonlinear) Wentzell boundary conditions (see Ref. 3). However, this work also uses the fact that the structure of the given boundary condition provides a nice monotonicity, which helps to get a semigroup in an appropriate Sobolev space. The nature of our model is very different than those in Refs. 11 and 3 due to the lack of monotonicity, since in our case $\lambda$ is real.

Our first aim in this paper is to study the blow-up problem in a more general context than in Ref. 1. In our model, the main equation also includes a nonlinear defocusing term $\left(k|u|^{p} u\right.$, $k>0$ ) and damping (iau, $a \geq 0$ ). In particular, we want to understand the nature of the competition between the bad term (nonlinear Robin boundary condition of focusing type) and the good terms (defocusing nonlinearity and damping). We show that there are solutions which blow up in finite time. More precisely, we prove that solutions cannot exist globally in $H^{1}$ sense if the initial data and powers of nonlinearities satisfy a certain set of conditions.

The second aim of this paper is to obtain decay rate estimates. We will prove exponential stabilization of solutions where the decay rates are determined according to the relation between the powers of the nonlinearities. We obtain different decay rates depending on the given relation between the powers of nonlinearities $r$ and $p$.

We comment on the critical exponent in the last chapter of the paper. Recall that the critical exponent in the case $k=0, a=0$ is $r^{*}=2$ (see Ref. 1). However, in the presence of the defocusing nonlinearity, we deduce that the critical exponent must also depend on $p$. For example, we show that every local solution is also global if $2 \leq r<\frac{p}{2}$ in Proposition 4.4. This shows that sufficiently strong defocusing nonlinearity in the main equation has a dominating effect on the nonlinear boundary condition.

Remark 1.1. We do not study the local well-posedness of (1). We assume that (1) has a unique classical local solution on a maximal time interval $\left[0, T_{\max }\right)\left(0<T_{\max } \leq \infty\right)$, which lies in a Sobolev space of sufficiently high order and also satisfies the blow-up alternative in $H^{1}$ sense: either $T_{\max }=\infty$ or else $T_{\max }<\infty$ and $\left\|u_{x}(t)\right\|_{L^{2}(I)} \rightarrow \infty$ as $t \uparrow T_{\max }$. For simplicity, we assume that the initial data are from $H^{s}\left(\mathbb{R}^{+}\right)$with s big enough and satisfy the necessary compatibility condition that guarantees the existence of a local classical solution. Indeed, the second author's recent paper ${ }^{4}$ proves the following local well-posedness theorem for the case $a=0$, but the proof can be trivially adapted to the case $a>0$. 
Theorem 1.2 (Local well-posedness). Let $T>0$ be arbitrary, $s \in\left(\frac{1}{2}, \frac{7}{2}\right)-\left\{\frac{3}{2}\right\}, p, r>0, k, \lambda \in$ $\mathbb{R}-\{0\}, u_{0} \in H^{s}\left(\mathbb{R}_{+}\right)$together with $u_{0}^{\prime}(0)=-\lambda\left|u_{0}(0)\right|^{r} u_{0}(0)$ whenever $s>\frac{3}{2}$. We in addition assume the following restrictions on $p$ and $r$.

(A1) If $s$ is integer, then $p \geq s$ if $p$ is an odd integer and $[p] \geq s-1$ if $p$ is non-integer.

(A2) If $s$ is non-integer, then $p>s$ if $p$ is an odd integer and $[p] \geq[s]$ if $p$ is non-integer.

(A3) $r>\frac{2 s-1}{4}$ if $r$ is an odd integer and $[r] \geq\left[\frac{2 s-1}{4}\right]$ if $r$ is non-integer.

Then, the following hold true.

(i) Local existence and uniqueness: There exists a unique local solution $u \in X_{T_{0}}^{s}$ of (1) for some $T_{0}=T_{0}\left(\left\|u_{0}\right\|_{H^{s}\left(\mathbb{R}_{+}\right)}\right) \in(0, T]$, where $X_{T_{0}}^{s}$ is the set of those elements in

$$
C\left(\left[0, T_{0}\right] ; H^{s}\left(\mathbb{R}_{+}\right)\right) \cap C\left(\mathbb{R}_{+}^{x} ; H^{\frac{2 s+1}{4}}\left(0, T_{0}\right)\right)
$$

that are bounded with respect to the norm $\|\cdot\|_{X_{T_{0}}^{s}}$. This norm is defined by

$$
\|u\|_{X_{T_{0}}^{s}}:=\sup _{t \in\left[0, T_{0}\right]}\|u(\cdot, t)\|_{H^{s}\left(\mathbb{R}_{+}\right)}+\sup _{x \in \mathbb{R}_{+}}\|u(x, \cdot)\|_{H^{\frac{2 s+1}{4}}\left(0, T_{0}\right)} .
$$

(ii) Continuous dependence: If $B$ is a bounded subset of $H^{s}\left(\mathbb{R}_{+}\right)$, then there is $T_{0}>0$ (depends on the diameter of $B$ ) such that the flow $u_{0} \rightarrow u$ is Lipschitz continuous from $B$ into $X_{T_{0}}^{s}$.

(iii) Blow-up alternative: If $S$ is the set of all $T_{0} \in(0, T]$ such that there exists a unique local solution in $X_{T_{0}}^{s}$, then whenever $T_{\max }:=\sup _{T_{0} \in S} T_{0}<T$, it must be true that $\lim _{t \uparrow T_{\max }}\|u(t)\|_{H^{s}\left(\mathbb{R}_{+}\right)}=\infty$.

\section{MAIN THEOREMS}

Here are our main results.

Theorem 2.1 (Blow-up). Suppose $r>\max \{2, p-2\}, E(0) \leq 0$, and

$$
\frac{(a-b)}{2} \int_{0}^{\infty} x^{2}\left|u_{0}(x)\right|^{2} d x<\operatorname{Im} \int_{0}^{\infty} x u_{0}(x)^{\prime} \bar{u}_{0}(x) d x,
$$

where $b=\frac{a(r+2)(4-M)}{4(r+2)-2 M}<0, M=\max \{8,2 p\}$. Then, there exists $T>0$ such that the corresponding local solution $u$ of (1) (see Remark 1.1) satisfies

$$
\lim _{t \rightarrow T^{-}}\left\|u_{x}(t)\right\|_{L^{2}(I)}=\infty .
$$

Remark 2.2. Note that in the case $a=0$, assumption (3) reduces to

$$
\operatorname{Im} \int_{0}^{\infty} x u_{0}^{\prime} \bar{u}_{0} d x>0 .
$$

This is the same assumption on the initial data in the context of the classical paper. ${ }^{9}$

Remark 2.3. Note that we do not assume that the initial energy is strictly negative. In the case $E(0)=0$, solutions do not have to blow-up if one disregards (3), e.g., the zero solution. As we will see in the proof, condition (3) forces solutions to blow-up in this case. However, if one puts a stronger assumption on the initial energy, such as strict negativeness in the case $a=0$, we believe that by using a compactly supported weight function, see, for example, Ref. 14, one might remove condition (3) and still obtain the blow-up in $H^{1}$ sense.

Theorem 2.4 (Stabilization). Suppose $u$ is a local solution of (1) (see Remark 1.1). Then we have the following.

(i) If $a>0, r<2$, then $u$ is global and

$$
\|u(t)\|_{H^{1}(I)}^{2} \leq C e^{-(2 a-\epsilon) t}, t \geq 0,
$$

where $\epsilon>0$ is fixed and small (can be chosen arbitrarily small), and $C=C\left(u_{0}, \epsilon, r\right)$ is a non-negative constant. 
(ii) If $a>0,2 \leq r<\frac{p}{2}$, then $u$ is global and

$$
\|u(t)\|_{H^{1}(I)}^{2} \leq C e^{-(a \mu-\epsilon) t}, t \geq 0,
$$

where

$$
\mu=\frac{(p+2)(p-2 r)}{p(p+2)-2 r},
$$

and $\epsilon>0$ is fixed and small (can be chosen arbitrarily small), and $C=C\left(u_{0}, \epsilon, r, p\right)$ is a non-negative constant.

(iii) if $a>0, r=2, p \leq 4$, and $u_{0}$ is sufficiently small in $L^{2}$ sense, then $u$ is global and

$$
\|u(t)\|_{H^{1}(I)}^{2} \leq C e^{-2 a t}, t \geq 0,
$$

where $C=C\left(u_{0}, p\right)$ is a non-negative constant.

(iv) if $a>0, r>2, r \geq \frac{p}{2}$, and $u_{0}$ is sufficiently small in $H^{1} \cap L^{p+2}$ sense, then $u$ is global and

$$
\|u(t)\|_{H^{1}(I)}^{2} \leq C e^{-2 a t}, t \geq 0,
$$

where $C=C\left(u_{0}, r, p\right)$ is a non-negative constant.

Remark 2.5. The following problem remains open.

- Is it possible to construct blow up solutions in the two cases $r=2, p \leq 4$ and $r>2, p-2 \geq$ $r \geq \frac{p}{2}$ ?

In our analysis, we show that this is not possible whenever we choose small enough initial data. However, this does not mean one cannot construct blow-up solutions with arbitrary initial data. An answer to the above problem will also help to determine the critical exponent for our model, see Section IV.

We summarize our results in Table I.

\section{BLOW-UP SOLUTIONS: PROOF OF THEOREM 2.1}

\section{A. Case $a \neq 0$}

\begin{tabular}{|c|c|c|c|}
\hline Nonlinear powers & Blow-up $(a \geq 0)$ & Local $\Rightarrow$ Global $(a \geq 0)$ & Exp. stabilization $(a>0)$ \\
\hline $\mathrm{r}<2$ & NO & YES & $\begin{array}{l}\text { YES } \\
\text { Decay rate } \sim O\left(e^{-(2 a-\epsilon) t}\right)\end{array}$ \\
\hline $2 \leq r<\frac{p}{2}$ & NO & YES & $\begin{array}{l}\text { YES } \\
\text { Decay rate } \sim O\left(e^{-(a \mu-\epsilon) t}\right)(\text { See (4)) }\end{array}$ \\
\hline \multirow[t]{2}{*}{$r=2, p \leq 4$} & & Small Sol. & $\begin{array}{l}\text { Small Sol. } \\
\text { Decay rate } \sim O\left(e^{-2 a t}\right)\end{array}$ \\
\hline & OPEN & Large Sol: OPEN & Large Sol: OPEN \\
\hline \multirow[t]{2}{*}{$r>2, p-2 \geq r \geq \frac{p}{2}$} & & Small Sol. & $\begin{array}{l}\text { Small Sol. } \\
\text { Decay rate } \sim O\left(e^{-2 a t}\right)\end{array}$ \\
\hline & OPEN & Large Sol: OPEN & Large Sol:OPEN \\
\hline \multirow[t]{2}{*}{$r>2, r>p-2$} & & ONLY Small Sol. & $\begin{array}{l}\text { ONLY Small Sol. } \\
\text { Decay rate } \sim O\left(e^{-2 a t}\right)\end{array}$ \\
\hline & YES & & \\
\hline
\end{tabular}

In this section, we prove Theorem 2.1 for the case $a \neq 0$, slightly modifying the proof in Ref. 22.

TABLE I. Blow-up, local/global solutions, and stabilization. 
Lemma 3.1. Let u be a local solution of (1) (see Remark 1.1) and $b \in \mathbb{R}$. Then,

(i) $\|u(t)\|_{L^{2}(I)}^{2}=e^{-2 a t}\left\|u_{0}\right\|_{L^{2}(I)}^{2}$,

(ii) $E(t) e^{2 b t}=E(0)+\int_{0}^{t} e^{2 b s} \rho(s) d s$

for $T_{0}>t \geq 0$, where $\rho$ is given by (8), and $E(t)$ is defined in (2).

Proof. We multiply (1) by $\bar{u}$, take the imaginary parts, integrate over $I \equiv(0, \infty)$, and obtain the exponential decay of the $L^{2}$-norm (conservation when $a=0$ ) of the solution,

$$
\frac{1}{2} \frac{d}{d t}\|u(t)\|_{L^{2}(I)}^{2}=-a\|u(t)\|_{L^{2}(I)}^{2} \Rightarrow\|u(t)\|_{L^{2}(I)}^{2}=e^{-2 a t}\left\|u_{0}(x)\right\|_{L^{2}(I)}^{2} .
$$

Now, we multiply (1) by $\bar{u}_{t}$, take two real parts, integrate the obtained relation over $I$, and get

$$
\begin{aligned}
\frac{d}{d t}\left(\left\|u_{x}(t)\right\|_{L^{2}(I)}^{2}\right. & \left.-\frac{2 \lambda}{r+2}|u(0, t)|^{r+2}+\frac{2 k}{p+2}\|u(t)\|_{L^{p+2}(I)}^{p+2}\right) \\
= & 2 \operatorname{Re} \int_{0}^{\infty} i a \bar{u} u_{t} d x=2 a \operatorname{Re} \int_{0}^{\infty} \bar{u}(x, t)\left(u_{x x}-k|u|^{p} u-i a u\right) d x \\
& =-2 a\left(\left\|u_{x}(t)\right\|_{L^{2}(I)}^{2}-\lambda|u(0, t)|^{r+2}+k\|u(t)\|_{L^{p+2}(I)}^{p+2}\right) \\
= & -2 a\left(\left\|u_{x}(t)\right\|_{L^{2}(I)}^{2}-\frac{2 \lambda}{r+2}|u(0, t)|^{r+2}+\frac{2 k}{p+2}\|u(t)\|_{L^{p+2}(I)}^{p+2}\right) \\
& -\frac{2 a k p}{p+2}\|u(t)\|_{L^{p+2}(I)}^{p+2}+\frac{2 a \lambda r}{r+2}|u(0, t)|^{r+2}
\end{aligned}
$$

Then, the identity in (5) is simply

$$
E^{\prime}(t)=-2 a E(t)-\frac{2 a k p}{p+2}\|u(t)\|_{L^{p+2}(I)}^{p+2}+\frac{2 a \lambda r}{r+2}|u(0, t)|^{r+2} .
$$

Adding $2 b E(t)$ to both sides, where $b \in \mathbb{R}$ and $b<a$, we have

$$
E^{\prime}(t)+2 b E(t)=(2 b-2 a) E(t)-\frac{2 a k p}{p+2}\|u(t)\|_{L^{p+2}(I)}^{p+2}+\frac{2 a \lambda r}{r+2}|u(0, t)|^{r+2} .
$$

Rewriting the right hand side of (7) by using the definition of $E(t)$, we have

$$
\begin{aligned}
E^{\prime}(t)+2 b E(t)=-(2 a-2 b)\left\|u_{x}(t)\right\|_{L^{2}(I)}^{2} & -\frac{4 \lambda b}{r+2}|u(0, t)|^{r+2} \\
& +\frac{4 k b}{p+2}\|u(t)\|_{L^{p+2}(I)}^{p+2}+2 a \lambda|u(0, t)|^{r+2}-2 a k\|u(t)\|_{L^{p+2}(I)}^{p+2} .
\end{aligned}
$$

Multiplying both sides by $e^{2 b t}$ and integrating over $(0, t)$, we have

$$
E(t) e^{2 b t}=E(0)+\int_{0}^{t} e^{2 b s} \rho(s) d s,
$$

where

$$
\begin{aligned}
\rho(t)=-(2 a-2 b)\left(\left\|u_{x}(t)\right\|_{L^{2}(I)}^{2}-\left(\frac{a(r+2)-2 b}{2 a-2 b}\right)\right. & \frac{2 \lambda}{r+2}|u(0, t)|^{r+2} \\
& \left.+\left(\frac{a(p+2)-2 b}{2 a-2 b}\right) \frac{2 k}{p+2}\|u(t)\|_{L^{p+2}(I)}^{p+2}\right) .
\end{aligned}
$$

Let us set

$$
\theta(t) \equiv\left\|u_{x}(t)\right\|_{L^{2}(I)}^{2}-\left(\frac{a(r+2)-2 b}{2 a-2 b}\right) \frac{2 \lambda}{r+2}|u(0, t)|^{r+2}+\frac{2 k}{p+2}\|u(t)\|_{L^{p+2}(I)}^{p+2} .
$$

Note that $\frac{a(r+2)-2 b}{2 a-2 b} \geq 1$, which implies $\theta(t) \leq E(t)$. Therefore, $\frac{a(p+2)-2 b}{2 a-2 b}-1=\frac{a p}{2 a-2 b}>0$, and by Lemma 3.1 


$$
\begin{array}{r}
\theta(t) e^{2 b t} \leq E(t) e^{2 b t}=E(0)-(2 a-2 b) \int_{0}^{t}\left(\theta(s)+\left(\frac{a p}{2 a-2 b}\right) \frac{2 k}{p+2}\|u(s)\|_{L^{p+2}(I)}^{p+2}\right) e^{2 b s} d s \\
\leq E(0)-(2 a-2 b) \int_{0}^{t} \theta(s) e^{2 b s} d s
\end{array}
$$

Multiplying (10) by $e^{(2 a-2 b) t}$, we get

$$
\frac{d}{d t}\left(e^{(2 a-2 b) t} \int_{0}^{t} e^{2 b s} \theta(s) d s\right) \leq E(0) e^{(2 a-2 b) t}
$$

from which it follows that

$$
\int_{0}^{t} \theta(s) e^{2 b s} d s \leq 0
$$

provided that $E(0) \leq 0$.

Now, we set

$$
I(t)=\int_{0}^{\infty} x^{2}|u|^{2} d x, V(t)=-4 \operatorname{Im} \int_{0}^{\infty} \bar{u} x u_{x} d x \text {, and } y(t)=-\frac{1}{4} V(t) .
$$

We have the following lemma.

\section{Lemma 3.2. I and y satisfy the following identities:}

(i) $e^{2 b t} I(t)+(2 a-2 b) \int_{0}^{t} e^{2 b s} I(s) d s=I(0)+\int_{0}^{t} V(s) e^{2 b s} d s$,

(ii) $\dot{y}+2 a y=-\frac{1}{4} \theta_{1}$, and

(iii) $V(t) e^{2 b t}=V(0)+(2 b-2 a) \int_{0}^{t} V(s) e^{2 b s} d s+\int_{0}^{t} \theta_{1}(s)^{2 b s} d s$

for $T_{0} \geq t \geq 0$, where $\theta_{1}$ is given in (27).

Proof. Differentiating $I(t)$, we have

$$
\begin{aligned}
\frac{d}{d t} I(t) & =\int_{0}^{\infty} x^{2}\left(u \bar{u}_{t}+u_{t} \bar{u}\right) d x=2 \operatorname{Re} \int_{0}^{\infty} x^{2} u_{t} \bar{u} d x \\
& =2 \operatorname{Im} \int_{0}^{\infty}\left(u_{x x}-k|u|^{p} u-i a u\right) x^{2} \bar{u} d x=-2 \operatorname{Im} \int_{0}^{\infty}\left(x^{2} \bar{u}\right)_{x} u_{x} d x-2 a \int_{0}^{\infty} x^{2}|u|^{2} d x \\
& =-4 \operatorname{Im} \int_{0}^{\infty} \bar{u} x u_{x} d x-2 a \int_{0}^{\infty} x^{2}|u|^{2} d x .
\end{aligned}
$$

Therefore,

$$
I^{\prime}(t)+2 a I(t)=-4 \operatorname{Im} \int_{0}^{\infty} \bar{u} x u_{x} d x
$$

Adding $2 b I(t)$ to both sides,

$$
I^{\prime}(t)+2 b I(t)=-(2 a-2 b) I(t)+V(t)
$$

Multiplying both sides by $e^{2 b t}$,

$$
\left(I(t) e^{2 b t}\right)^{\prime}=-(2 a-2 b) I(t) e^{2 b t}+V(t) e^{2 b t} .
$$

Integrating over $(0, t)$, we have

$$
e^{2 b t} \int_{0}^{\infty} x^{2}|u|^{2} d x+(2 a-2 b) \int_{0}^{t} e^{2 b s} \int_{0}^{\infty} x^{2}|u|^{2} d x d s=\int_{0}^{\infty} x^{2}\left|u_{0}\right|^{2} d x+\int_{0}^{t} V(s) e^{2 b s} d s .
$$

Differentiating $y(t)$, we have

$$
\frac{d}{d t} y(t)=\frac{d}{d t} \operatorname{Im} \int_{0}^{\infty} \bar{u} x u_{x} d x=\operatorname{Im} \int_{0}^{\infty}\left(\bar{u}_{t} x u_{x}+\bar{u} x u_{x t}\right) d x .
$$


Integrating by parts we obtain

$$
\operatorname{Im} \int_{0}^{\infty} \bar{u} x u_{x t} d x=-\operatorname{Im} \int_{0}^{\infty}(\bar{u} x)_{x} u_{t} d x=-\operatorname{Im} \int_{0}^{\infty} \bar{u}_{x} x u_{t} d x-\operatorname{Im} \int_{0}^{\infty} \bar{u} u_{t} d x .
$$

Hence,

$$
\frac{d}{d t} y(t)=2 \operatorname{Im} \int_{0}^{\infty} \bar{u}_{t} x u_{x} d x-\operatorname{Im} \int \bar{u} u_{t} d x .
$$

The first term on the right hand side of (21) is

$$
\begin{aligned}
2 \operatorname{Im} \int_{0}^{\infty} \bar{u}_{t} x u_{x} d x=2 \operatorname{Im} & \int_{0}^{\infty}\left(i \bar{u}_{x x}-i k|u|^{p} \bar{u}-a \bar{u}\right) x u_{x} d x \\
& =2 \operatorname{Re} \int_{0}^{\infty} \bar{u}_{x x} x u_{x} d x-2 \operatorname{Re} \int_{0}^{\infty} k x|u|^{p} \bar{u} u_{x} d x-2 a \operatorname{Im} \int_{0}^{\infty} x \bar{u} u_{x} d x,
\end{aligned}
$$

where

$$
2 \operatorname{Re} \int_{0}^{\infty} \bar{u}_{x x} x u_{x} d x=\operatorname{Re} \int_{0}^{\infty} x\left(\left|u_{x}\right|^{2}\right)_{x} d x=-\int_{0}^{\infty}\left|u_{x}\right|^{2} d x
$$

and

$$
\begin{aligned}
-2 \operatorname{Re} \int_{0}^{\infty} k x|u|^{p} \bar{u} u_{x} d x=-\frac{2 k}{p+2} \operatorname{Re} \int_{0}^{\infty} x\left(|u|^{p+2}\right)_{x} d x & \\
& =\frac{2 k}{p+2} \int_{0}^{\infty}|u|^{p+2} d x=\frac{2 k}{p+2}\|u\|_{L^{p+2}(I)}^{p+2} .
\end{aligned}
$$

The second term on the right hand side of (21) is

$$
\begin{aligned}
-\operatorname{Im} \int_{0}^{\infty} \bar{u} u_{t} d x=-\operatorname{Im} \int_{0}^{\infty} \bar{u}\left(-i u_{x x}+i k|u|^{p} u-a u\right) d x \\
=\operatorname{Re} \int_{0}^{\infty}\left(\bar{u} u_{x x} d x-k\|u\|_{L^{p+2}(I)}^{p+2}\right) d x \\
\quad=-\int_{0}^{\infty}\left(\left|u_{x}\right|^{2} d x+\lambda|u(0, t)|^{r+2}-k\|u\|_{L^{p+2}(I)}^{p+2}\right) d x .
\end{aligned}
$$

Combining (21)-(25), we obtain

$$
\frac{d}{d t} y(t)=-2\left\|u_{x}\right\|_{L^{2}(I)}^{2}-\frac{k p}{p+2}\|u\|_{L^{p+2}(I)}^{p+2}+\lambda|u(0, t)|^{r+2}-2 a \operatorname{Im} \int_{0}^{\infty} x \bar{u} u_{x} d x .
$$

Multiplying (26) by -4 and rearranging the terms, we have

$$
\frac{d}{d t} V(t)+2 a V(t)=8\left\|u_{x}\right\|_{L^{2}(I)}^{2}+\frac{4 k p}{p+2}\|u\|_{L^{p+2}(I)}^{p+2}-4 \lambda|u(0, t)|^{r+2} \equiv \theta_{1}(t) .
$$

Adding $(2 b-2 a) V(t)$ to both sides of (27), multiplying the obtained relation by $e^{2 b t}$ and integrating over the interval $(0, t)$, we obtain

$$
V(t) e^{2 b t}=V(0)+(2 b-2 a) \int_{0}^{t} V(s) e^{2 b s} d s+\int_{0}^{t} \theta_{1}(s) e^{2 b s} d s
$$

Let $M=\max \{8,2 p\}$ and $b=\frac{a(r+2)(4-M)}{4(r+2)-2 M}$, then $b<0$ since $r>\max \{2, p-2\}$, and moreover

$$
-M\left(\frac{a(r+2)-2 b}{2 a-2 b}\right) \frac{2 \lambda}{r+2}|u(0, t)|^{r+2} \geq-4 \lambda|u(0, t)|^{r+2} .
$$

On the other hand, $M\left\|u_{x}\right\|^{2} \geq 8\left\|u_{x}\right\|^{2}$ and

$$
M \frac{2 k}{p+2}\|u\|_{L^{p+2(I)}}^{p+2} \geq \frac{4 k p}{p+2}\|u\|_{L^{p+2}(I)}^{p+2}
$$


Therefore, $\theta_{1}(t) \leq \theta(t)$, and by (12) and (28),

$$
V(t) e^{2 b t} \leq V(0)+(2 b-2 a) \int_{0}^{t} V(s) e^{2 b s} d s,
$$

which can also be written as

$$
\frac{d}{d t}\left(e^{(2 a-2 b) t} \int_{0}^{t} V(s) e^{2 b s} d s\right) \leq V(0) e^{(2 a-2 b) t} .
$$

Integrating (30) over $(0, t)$, we obtain

$$
\int_{0}^{t} V(s) e^{2 b s} d s \leq \frac{1}{2 a-2 b}\left(1-e^{-(2 a-2 b) t}\right) V(0) .
$$

From this inequality, one obtains the blow-up of the solutions. Indeed, let

$$
z(t) \equiv e^{2 b t} \int_{0}^{\infty} x^{2}|u|^{2} d x .
$$

Then by (18),

$$
z(t) \leq \int_{0}^{\infty} x^{2}\left|u_{0}\right|^{2} d x+\frac{1}{2 a-2 b}\left(1-e^{-(2 a-2 b) t}\right) V(0) .
$$

Hence,

$$
\lim _{t \rightarrow T} z(t)=0,
$$

where $T \equiv-\frac{1}{2 a-2 b} \ln \left(\frac{(2 a-2 b) \int x^{2}\left|u_{0}\right|^{2} d x+V(0)}{V(0)}\right)$. We choose $u_{0}$ in such a way that $T>0$ by assumption (3). Now, using the decay of the $L^{2}$ norm which was proved in Lemma 3.1, we deduce the inequality

$$
\|u(t)\|_{L^{2}(I)}^{2}=-2 \operatorname{Re} \int_{0}^{\infty} x u \bar{u}_{x} d x \leq 2\|x u(x, t)\|_{L^{2}(I)} \cdot\left\|u_{x}(t)\right\|_{L^{2}(I)} .
$$

The last inequality implies

$$
\left\|u_{x}(t)\right\|_{L^{2}(I)} \geq \frac{\left\|u_{0}(x)\right\|_{L^{2}(I)}^{2} e^{-(2 a-2 b) t}}{z(t)} \rightarrow \infty
$$

as $t \rightarrow T$.

\section{B. Case $a=0$}

In this section, we prove Theorem 2.1 for $a=0$ by obtaining a nonlinear ordinary differential inequality which yields blow-up of solutions. The proof follows by adapting the same argument in Ref. 9 to our model.

$$
\frac{1}{2}\left\|u_{x}(t)\right\|_{L^{2}(I)}^{2}+\frac{k}{p+2}\|u(t)\|_{L^{p+2}(I)}^{p+2}=E(0)+\frac{\lambda}{r+2}|u(0, t)|^{r+2} .
$$

Then,

$$
\frac{r+2}{2}\left\|u_{x}(t)\right\|_{L^{2}(I)}^{2}+\frac{k(r+2)}{p+2}\|u(t)\|_{L^{p+2}(I)}^{p+2} \leq \lambda|u(0, t)|^{r+2}
$$

provided that $E(0) \leq 0$,

$$
y^{\prime}(t) \geq\left(\frac{r-2}{2}\right)\left\|u_{x}\right\|_{L^{2}(I)}^{2}+\frac{k(r-p+2)}{p+2}\|u(t)\|_{L^{p+2(I)}}^{p+2} .
$$

Then $y^{\prime}(t) \geq \kappa\left\|u_{x}(t)\right\|_{L^{2}(I)}^{2}$ for some $\kappa>0$ provided that $r>\max \{2, p-2\}$. Therefore $y(t)>0$, since $y(0)>0$. This means $I^{\prime}(t)=-4 y(t) \leq 0$. Hence, $I(t) \leq I(0)$. By definition of $y(t)$, we have $|y(t)| \leq \sqrt{I(0)}\left\|u_{x}\right\|_{L^{2}(I)}$. Hence, $y^{\prime}(t) \geq \kappa \frac{y^{2}(t)}{I(0)}$. Separating the variables and integrating this differential inequality over the interval $(0, t)$, and using $y(0)>0$, we get 


$$
\int_{0}^{t} \frac{d y}{y^{2}}=\int_{0}^{t} \frac{\kappa}{I(0)} d s \Rightarrow y(t) \geq \frac{y(0) I(0)}{I(0)-\kappa y(0) t}
$$

That is to say,

$$
\left\|u_{x}\right\|_{L^{2}(I)} \geq \frac{y(t)}{\sqrt{I(0)}} \geq \frac{y(0) \sqrt{I(0)}}{I(0)-\kappa y(0) t} .
$$

Hence, we deduce that

$$
\lim _{t \rightarrow T^{-}}\left\|u_{x}(t)\right\|_{L^{2}(I)}=\infty
$$

where $T \equiv \frac{I(0)}{\kappa y(0)}$.

\section{CRITICAL EXPONENT AND EXPONENTIAL DECAY ESTIMATES}

\section{A. Critical exponent conjecture}

It is not difficult to obtain uniform boundedness (in time variable) of the $H^{1}$ norm if $r<2$ for arbitrarily large initial data and if $r=2, p \leq 4$ for small initial data. In order to prove this, one can simply proceed as in Ref. 1 for $a=0$. Regarding the damped situation $(a>0)$, see Section IV B below. However, we expect that the situation in our model should be better than this due to the defocusing source term $k|u|^{p} u, k>0$. We conjecture that if $p>4$, then one can control the $H^{1}$ norm of the solutions with arbitrarily large initial data, even if $2 \leq r<p-2$. In addition, one should be able to control the $H^{1}$ norm with small data for $r \geq p-2$ whenever $p>4$. More precisely, we have the following conjecture.

Conjecture 4.1. The critical exponent for nonlinear model (1) is

$$
r^{*}=\max \{2, p-2\} \text {. }
$$

One can try to use interpolation on $L^{p}$-spaces to obtain some partial results. Let us assume $a=0$ for simplicity. Observe that

$$
\left\|u_{x}(t)\right\|_{L^{2}(I)}^{2}+\frac{2 k}{p+2}\|u(t)\|_{p+2}^{p+2} \leq|E(0)|+\frac{2 \lambda}{r+2}|u(0, t)|^{r+2} .
$$

By $\epsilon$-Young's inequality and Hölder's inequality,

$$
\begin{array}{r}
\frac{2 \lambda}{r+2}|u(0, t)|^{r+2}=-\frac{2 \lambda}{r+2} \int_{0}^{\infty}\left(|u|^{r+2}\right)_{x} d x=-2 \lambda \operatorname{Re} \int_{0}^{\infty}|u|^{r} u \bar{u}_{x} d x \\
\leq \epsilon\left\|u_{x}\right\|_{L^{2}(I)}^{2}+C_{\epsilon} \int_{0}^{\infty}|u|^{2 r+2} d x=\epsilon\left\|u_{x}\right\|_{L^{2}(I)}^{2}+C_{\epsilon} \int_{0}^{\infty}|u|^{2 r+2-\delta}|u|^{\delta} d x \\
\leq \epsilon\left\|u_{x}\right\|_{L^{2}(I)}^{2}+C_{\epsilon}\|u\|_{L^{2}(I)}^{\frac{\delta}{2}}\|u\|_{\frac{2(2 r+2-\delta)}{2-\delta}}^{\frac{2-\delta}{2}},
\end{array}
$$

where $\epsilon>0$ is fixed and can be chosen arbitrarily small.

If we choose $\delta=2-\frac{4 r}{p}$, which is positive if $p>2 r$, use the mass identity (mass is conserved if $a=0$ ), and Hölder's inequality again, then we obtain

$$
\begin{aligned}
|u(0, t)|^{r+2} \leq \epsilon\left\|u_{x}(t)\right\|_{L^{2}(I)}^{2}+C_{\epsilon}\|u(t)\|_{L^{2}(I)}^{\frac{p-2 r}{p}}\|u(t)\|_{p+2}^{\frac{2 r}{p}} \leq \epsilon\left\|u_{x}(t)\right\|_{L^{2}(I)}^{2} & \\
& +C_{\epsilon}\left\|u_{0}\right\|_{L^{2}(I)}^{\frac{(p+2)(p-2 r)}{p(p+2 r}}+\frac{2 k \epsilon}{p+2}\|u(t)\|_{p+2}^{p+2} .
\end{aligned}
$$

Using this in (31), we get

$$
(1-\epsilon)\left(\left\|u_{x}(t)\right\|_{L^{2}(I)}^{2}+\frac{2 k}{p+2}\|u(t)\|_{p+2}^{p+2}\right) \leq|E(0)|+C_{\epsilon}\left\|u_{0}\right\|_{L^{2}(I)}^{\frac{(p+2)(p-2 r)}{p(I)-2 r}}
$$

Hence we have $\left\|u_{x}\right\|_{L^{2}(I)} \leq C$ for some $C>0$. 
One can improve the above analysis by involving the case $r>2, r \geq \frac{p}{2}$ under a smallness assumption on the initial data. Indeed, by (31) and (37), we have

$$
\left\|u_{x}(t)\right\|_{L^{2}(I)}^{2} \leq\left\|u_{0}^{\prime}\right\|_{L^{2}(I)}^{2}+\frac{2 k}{p+2}\left\|u_{0}\right\|_{p+2}^{p+2}+\frac{2^{\frac{r+4}{2}} \lambda}{r+2}\left\|u_{0}\right\|_{L^{2}(I)}^{\frac{r+2}{2}}\left\|u_{x}\right\|_{L^{2}(I)}^{\frac{r+2}{2}} .
$$

If we set $\Phi(t) \equiv\left\|u_{x}(t)\right\|_{L^{2}(I)}^{2}$, then (33) can be rewritten as

$$
\Phi(t) \leq C_{1}+C_{2} \Phi(t)^{\sigma}
$$

where

$$
C_{1} \equiv\left\|u_{0}^{\prime}\right\|_{L^{2}(I)}^{2}+\frac{2 k}{p+2}\left\|u_{0}\right\|_{p+2}^{p+2}, C_{2} \equiv \frac{2^{\frac{r+4}{2}} \lambda}{r+2}\left\|u_{0}\right\|_{L^{2}(I)}^{\frac{r+2}{2}}
$$

and $\sigma \equiv \frac{r+2}{4}>1$. Since, $\Phi(0) \leq C_{1}$, then for sufficiently small $u_{0}$, one can have $C_{1} C_{2}^{\frac{1}{\sigma-1}} \leq \frac{\sigma-1}{\sigma \frac{\sigma}{\sigma-1}}$, we conclude that $\Phi(t) \leq \frac{\sigma}{\sigma-1} C_{1}$. For a justification of the smallness argument we carried out, we use the following lemma.

Lemma 4.2 (Ref. 19). Suppose

$$
\Phi(t) \leq C_{1}+C_{2} \Phi(t)^{\sigma}, \forall t \in[0, T),
$$

where $\Phi:[0, T) \rightarrow \mathbb{R}$ is non-negative, continuous, $C_{i}>0(i=1,2), \sigma>1$, and $\gamma=\frac{1}{\sigma-1}$. If $\Phi(0) \leq$ $C_{1}$ and $C_{1} C_{2}^{\gamma} \leq(\sigma-1) \sigma^{-\gamma-1}$. Then

$$
\Phi(t) \leq \frac{\sigma}{\sigma-1} C_{1}, \quad \forall t \in[0, T) .
$$

\section{B. Effect of damping: Proof of Theorem 2.4}

Our analysis above shows that although it is more difficult to prove the blow-up result in the presence of the damping term iau, $a>0$, damping actually plays no particular role in the blow-up condition $r>\max \{2, p-2\}$. This is analogous to the result in Ref. 22. Nevertheless, damping may have a stabilizing effect in the case that global solutions exist. See, for example, Ref. 21. For our model, this is easy to show in the case $r<2$ but is difficult to show if $2 \leq r<p-2$ whenever $p>4$, as in Section IV A.

Indeed, by Lemma 3.1, we have

$$
\begin{array}{r}
|u(0, t)|^{2}=-\int_{0}^{\infty}\left(|u|^{2}\right)_{x} d x=-2 \operatorname{Re} \int_{0}^{\infty} u \bar{u}_{x} d x \\
\quad \leq 2\|u\|_{L^{2}(I)}\left\|u_{x}\right\|_{L^{2}(I)} \leq 2\left\|u_{0}\right\|_{L^{2}(I)} e^{-a t}\left\|u_{x}\right\|_{L^{2}(I)},
\end{array}
$$

which implies

$$
|u(0, t)|^{r+2} \leq 2^{\frac{r+2}{2}}\left\|u_{0}\right\|_{L^{2}(I)}^{\frac{r+2}{2}} e^{-a \frac{(r+2)}{2} t}\left\|u_{x}\right\|_{L^{2}(I)}^{\frac{r+2}{2}} .
$$

Now, if $r<2$, then by $\epsilon$-Young's inequality, the right hand side of the above inequality is bounded by

$$
C_{\epsilon} e^{-a \mu t}+\epsilon\left\|u_{x}\right\|_{L^{2}(I)}^{2}
$$

where $\epsilon, C_{\epsilon}>0$ (generic constants) and $\mu=\frac{2(r+2)}{2-r}$. Observe that $\mu=2+\frac{4 r}{2-r}>2$. Multiplying identity (6) by $e^{2 a t}$ and integrating over the time interval $(0, t)$,

$$
E(t) e^{2 a t}=E(0)-\frac{2 a k p}{p+2} \int_{0}^{t}\|u(s)\|_{L^{p+2}(I)}^{p+2} e^{2 a s} d s+\frac{2 a \lambda r}{r+2} \int_{0}^{t}|u(0, s)|^{r+2} e^{2 a s} d s,
$$

which gives 


$$
\begin{gathered}
\left\|u_{x}\right\|_{L^{2}(I)}^{2} e^{2 a t} \leq \frac{2 \lambda}{r+2}|u(0, t)|^{r+2} e^{2 a t}-\frac{2 k}{p+2}\|u(t)\|_{L^{p+2}(I)}^{p+2} e^{2 a t} \\
+E(0)-\frac{2 a k p}{p+2} \int_{0}^{t}\|u(s)\|_{L^{p+2}(I)}^{p+2} e^{2 a s} d s+\frac{2 a \lambda r}{r+2} \int_{0}^{t}|u(0, s)|^{r+2} e^{2 a s} d s \\
\quad \leq \frac{2 \lambda}{r+2}|u(0, t)|^{r+2} e^{2 a t}+E(0)+\frac{2 a \lambda r}{r+2} \int_{0}^{t}|u(0, s)|^{r+2} e^{2 a s} d s \\
\leq C_{\epsilon} e^{a(2-\mu) t}+\epsilon\left\|u_{x}\right\|_{L^{2}(I)}^{2} e^{2 a t}+|E(0)|+\int_{0}^{t} C_{\epsilon} e^{a(2-\mu) s} d s+\epsilon \int_{0}^{t}\left\|u_{x}\right\|_{L^{2}(I)}^{2} e^{2 a s} d s
\end{gathered}
$$

which implies

$$
\left\|u_{x}\right\|_{L^{2}(I)}^{2} e^{2 a t} \leq C_{\epsilon}+\epsilon \int_{0}^{t}\left\|u_{x}\right\|_{L^{2}(I)}^{2} e^{2 a s} d s
$$

By Gronwall's lemma,

$$
\left\|u_{x}\right\|_{L^{2}(I)}^{2} e^{2 a t} \leq C_{\epsilon} e^{\epsilon t} \Rightarrow\left\|u_{x}\right\|_{L^{2}(I)}^{2} \leq C_{\epsilon} e^{-(2 a-\epsilon) t} .
$$

Combining the above result with the $L^{2}$ decay (see Lemma 3.1), we obtain the following result.

Proposition 4.3 (Stabilization I). Let $a>0, r<2$ and $u$ be a local solution of (1) (see Remark 1.1). Then $u$ is global and decays to zero exponentially fast in the following sense:

$$
\|u(t)\|_{H^{1}(I)}^{2} \leq C e^{-(2 a-\epsilon) t}, t \geq 0,
$$

where $\epsilon>0$ is fixed and can be chosen arbitrarily small.

Regarding the powers $r \geq 2$, one can also obtain similar decay estimates, but only under a smallness assumption on the initial data for some values of $p$.

Let us start with the case $2 \leq r<\frac{p}{2}$. By an argument similar to that in Section IV A, we have the following estimate:

$$
\frac{2 \lambda}{r+2}|u(0, t)|^{r+2} \leq \epsilon\left\|u_{x}\right\|_{L^{2}(I)}^{2}+C_{\epsilon}\left\|u_{0}\right\|_{L^{2}(I)}^{\mu} e^{-a \mu t}+\frac{2 k \epsilon}{p+2}\|u\|_{p+2}^{p+2}
$$

where

$$
\mu=\frac{(p+2)(p-2 r)}{p(p+2)-2 r}>0
$$

By (38), we have

$$
\begin{gathered}
\left\|u_{x}\right\|_{L^{2}(I)}^{2} e^{2 a t}+\frac{2 k}{p+2}\|u(t)\|_{L^{p+2}(I)}^{p+2} e^{2 a t} \leq \frac{2 \lambda}{r+2}|u(0, t)|^{r+2} e^{2 a t}- \\
+E(0)-\frac{2 a k p}{p+2} \int_{0}^{t}\|u(s)\|_{L^{p+2}(I)}^{p+2} e^{2 a s} d s+\frac{2 a \lambda r}{r+2} \int_{0}^{t}|u(0, s)|^{r+2} e^{2 a s} d s \\
\leq \frac{2 \lambda}{r+2}|u(0, t)|^{r+2} e^{2 a t}+E(0)+\frac{2 a \lambda r}{r+2} \int_{0}^{t}|u(0, s)|^{r+2} e^{2 a s} d s \\
\leq|E(0)|+C_{\epsilon} e^{a(2-\mu) t}+\int_{0}^{t} C_{\epsilon} e^{a(2-\mu) s} d s \\
+\epsilon\left(\left\|u_{x}\right\|_{L^{2}(I)}^{2}+\frac{2 k}{p+2}\|u\|_{p+2}^{p+2}\right) e^{2 a t}+\epsilon \int_{0}^{t}\left(\left\|u_{x}\right\|_{L^{2}(I)}^{2}+\frac{2 k}{p+2}\|u\|_{p+2}^{p+2}\right) e^{2 a s} d s
\end{gathered}
$$

Observe that

$$
\int_{0}^{t} C_{\epsilon} e^{a(2-\mu) s} d s=\frac{C_{\epsilon}}{a(2-\mu)}\left(e^{a(2-\mu) t}-1\right) \leq \frac{C_{\epsilon}}{a(2-\mu)} e^{a(2-\mu) t} .
$$

Let us set $\Psi(t) \equiv\left(\left\|u_{x}\right\|_{L^{2}(I)}^{2}+\frac{2 k}{p+2}\|u\|_{p+2}^{p+2}\right) e^{2 a t}$, then the above inequality reads

$$
(1-\epsilon) \Psi(t) \leq \alpha(t)+\epsilon \int_{0}^{t} \Psi(s) d s
$$


where $\alpha(t) \equiv|E(0)|+C_{\epsilon}\left(1+\frac{1}{a(2-\mu)}\right) e^{a(2-\mu) t}$. Note that $\alpha$ is a non-decreasing function since $\mu<2$. Now, by Gronwall's lemma, we have

$$
\Psi(t) \leq \frac{1}{1-\epsilon} \alpha(t) \exp \left(\frac{\epsilon t}{1-\epsilon}\right),
$$

which gives

$$
\left\|u_{x}\right\|_{L^{2}(I)}^{2} \leq C e^{-(a \mu-\epsilon) t}, t \geq 0 .
$$

This is a slower rate of decay than in Proposition 4.3. Hence, we proved the following proposition.

Proposition 4.4 (Stabilization II). Let $a>0,2 \leq r<\frac{p}{2}$ and $u$ be a local solution of (1) (see Remark 1.1). Then u is global and decays to zero exponentially fast in the following sense:

$$
\|u(t)\|_{H^{1}(I)}^{2} \leq C e^{-(a \mu-\epsilon) t}, t \geq 0,
$$

where $\mu$ is given by (42), and $\epsilon>0$ is fixed and can be chosen arbitrarily small.

Now, let us consider the case $r=2$ and $p \leq 4$. Using (37), we obtain

$$
|u(0, t)|^{4} \leq 2^{2}\left\|u_{0}\right\|_{L^{2}(I)}^{2} e^{-2 a t}\left\|u_{x}\right\|_{L^{2}(I)}^{2} .
$$

Now, by (38), we have

$$
\begin{aligned}
& \left\|u_{x}\right\|_{L^{2}(I)}^{2} e^{2 a t} \leq \lambda|u(0, t)|^{4} e^{2 a t}+|E(0)|+a \lambda \int_{0}^{t}|u(0, t)|^{4} e^{2 a s} d s \\
& \quad \leq|E(0)|+4 \lambda\left\|u_{0}\right\|_{L^{2}(I)}^{2} e^{-2 a t}\left\|u_{x}\right\|_{L^{2}(I)}^{2} e^{2 a t}+4 a \lambda\left\|u_{0}\right\|_{L^{2}(I)}^{2} \int_{0}^{t} e^{-2 a s}\left\|u_{x}\right\|_{L^{2}(I)}^{2} e^{2 a s} d s .
\end{aligned}
$$

Now, if we assume $\left\|u_{0}\right\|_{L^{2}(I)}^{2}<\frac{1}{4 \lambda}$, and since $e^{-2 a t} \leq 1$, we have

$$
\left(1-4 \lambda\left\|u_{0}\right\|_{L^{2}(I)}^{2}\right)\left\|u_{x}\right\|_{L^{2}(I)}^{2} e^{2 a t} \leq|E(0)|+4 a \lambda\left\|u_{0}\right\|_{L^{2}(I)}^{2} \int_{0}^{t} e^{-2 a s}\left\|u_{x}\right\|_{L^{2}(I)}^{2} e^{2 a s} d s,
$$

from which it follows that

$$
\left\|u_{x}\right\|_{L^{2}(I)}^{2} e^{2 a t} \leq \frac{|E(0)|}{1-4 \lambda\left\|u_{0}\right\|_{L^{2}(I)}^{2}}+\frac{4 a \lambda\left\|u_{0}\right\|_{L^{2}(I)}^{2}}{1-4 \lambda\left\|u_{0}\right\|_{L^{2}(I)}^{2}} \int_{0}^{t} e^{-2 a s}\left\|u_{x}\right\|_{L^{2}(I)}^{2} e^{2 a s} d s .
$$

Applying Gronwall's inequality to the above, we get

$$
\begin{aligned}
\left\|u_{x}\right\|_{L^{2}(I)}^{2} e^{2 a t} \leq \frac{|E(0)|}{1-4 \lambda\left\|u_{0}\right\|_{L^{2}(I)}^{2}} \exp \left(\frac{4 a \lambda\left\|u_{0}\right\|_{L^{2}(I)}^{2}}{1-4 \lambda\left\|u_{0}\right\|_{L^{2}(I)}^{2}} \int_{0}^{t} e^{-2 a s} d s\right) \\
\leq \frac{|E(0)|}{1-4 \lambda\left\|u_{0}\right\|_{L^{2}(I)}^{2}} \exp \left(\frac{2 \lambda\left\|u_{0}\right\|_{L^{2}(I)}^{2}}{1-4 \lambda\left\|u_{0}\right\|_{L^{2}(I)}^{2}}\right) .
\end{aligned}
$$

Hence, there exists $C>0$ such that $\left\|u_{x}(t)\right\|_{L^{2}(I)}^{2} \leq C e^{-2 a t}$ for $t \geq 0$. Therefore, we have proved the following result.

Proposition 4.5 (Stabilization III). Let $a>0, r=2, p \leq 4$ and $u$ be a local solution of (1) (see Remark 1.1) such that $u_{0}$ is sufficiently small in $L^{2}$ sense. Then $u$ is global and moreover $u$ decays to zero exponentially fast in the following sense:

$$
\|u(t)\|_{H^{1}(I)}^{2} \leq C e^{-2 a t}, t \geq 0 .
$$

Observe that the decay rate obtained in Proposition 4.5 is faster than the decay rates in Propositions 4.3 and 4.4 .

Now, let us consider the case $r>2, r \geq \frac{p}{2}$. 
By (38) and (37),

$$
\left\|u_{x}\right\|_{L^{2}(I)}^{2} e^{2 a t} \leq C_{1}+C_{2}\left\|u_{x}\right\|_{L^{2}(I)}^{\frac{r+2}{2}} e^{2 a t}+\operatorname{ar} C_{2} \int_{0}^{t} e^{-a\left(\frac{r+2}{2}\right) s}\left\|u_{x}\right\|_{L^{2}(I)}^{\frac{r+2}{2}} e^{2 a s} d s,
$$

where $C_{1}$ and $C_{2}$ are given in (35).

Let us define $S(t)=\sup _{[0, t]}\left\{\left\|u_{x}\right\|_{L^{2}(I)}^{2} e^{2 a s}\right\}$. Then since $\frac{r+2}{4}>1$, we have

$$
S(t) \leq C_{1}+C_{2} S(t)^{\frac{r+2}{4}}+a r C_{2} S(t)^{\frac{r+2}{4}} \int_{0}^{t} e^{-a\left(\frac{r+2}{2}\right) s} d s \leq C_{1}+\left(1+\frac{4 r}{r+2}\right) C_{2} S(t)^{\frac{r+2}{4}} .
$$

By the same smallness argument in (34) or Lemma 4.2, we obtain

$$
S(t) \leq \frac{2(r+2)}{r-2} C_{1}
$$

Hence, we proved the following proposition,

Proposition 4.6 (Stabilization IV). Let a $>0, r>2, r \geq \frac{p}{2}$, and $u$ be a local solution of (1) (see Remark 1.1) such that $u_{0}$ is sufficiently small in $H^{1} \cap L^{p+2}$ sense. Then $u$ is global and moreover $u$ decays to zero exponentially fast in the following sense:

$$
\|u(t)\|_{H^{1}(I)}^{2} \leq C e^{-2 a t}, t \geq 0 .
$$

\section{ACKNOWLEDGMENTS}

We are thankful for financial support from Izmir Institute of Technology through BAP Grant No. 2015IYYTE43.

${ }^{1}$ A. S. Ackleh and K. Deng, "On the critical exponent for the Schrödinger equation with a nonlinear boundary condition," Differ. Integr. Equations 17(11-12), 1293-1307 (2004).

${ }^{2}$ R. Balakrishnan, "Soliton propagation in nonuniform media," Phys. Rev. A 32(2), 1144-1149 (1985).

${ }^{3}$ M. Cavalcanti, W. Correa, I. Lasiecka, and C. Lefler, "Well-posedness and uniform stability for nonlinear Schrödinger equations with dynamic/wentzell boundary conditions," Indiana Univ. Math. J. (unpublished).

${ }^{4}$ A. Batal and T. Özsarı, "Nonlinear Schrödinger equation on the half-line with nonlinear boundary condition," e-print arXiv: 1507.04666 [math.AP]

${ }^{5}$ M. M. Cavalcanti, V. N. Domingos Cavalcanti, J. A. Soriano, and F. Natali, "Qualitative aspects for the cubic nonlinear Schrödinger equations with localized damping: Exponential and polynomial stabilization,” J. Differ. Equations 248(12), 2955-2971 (2010).

${ }^{6}$ C. A. Bortot, M. M. Cavalcanti, W. J. Corrêa, and V. N. Domingos Cavalcanti, "Uniform decay rate estimates for Schrödinger and plate equations with nonlinear locally distributed damping," J. Differ. Equations 254(9), 3729-3764 (2013).

${ }^{7}$ C. A. Bortot and M. M. Cavalcanti, "Asymptotic stability for the damped Schrödinger equation on noncompact Riemannian manifolds and exterior domains," Commun. Partial Differ. Equations 39(9), 1791-1820 (2014).

${ }^{8}$ G. Fibich, "Self-focusing in the damped nonlinear Schrödinger equation," SIAM J. Appl. Math. 61(5), 1680-1705 (2001).

${ }^{9}$ R. T. Glassey, “On the blowing up of solutions to the Cauchy problem for nonlinear Schrödinger equations," J. Math. Phys. 18(9), 1794-1797 (1977).

${ }^{10}$ A. V. Gurevich, Nonlinear Phonomena in the Ionosphere (Springer, 1978).

${ }^{11}$ I. Lasiecka and R. Triggiani, "Well-posedness and sharp uniform decay rates at the $L^{2}(\Omega)$-level of the Schrödinger equation with nonlinear boundary dissipation,” J. Evol. Equations 6(3), 485-537 (2006).

12 M. Ohta and G. Todorova, "Remarks on global existence and blowup for damped nonlinear Schrödinger equations," Discrete Contin. Dyn. Syst. 23(4), 1313-1325 (2009).

${ }^{13}$ B. A. Malomed, Nonlinear Schrödinger Equations (Encyclopedia of Nonlinear Science, New York, 2005).

${ }^{14}$ T. Ogawa and Y. Tsutsumi, "Blow-up of $H^{1}$ solutions for the one-dimensional nonlinear Schrödinger equation with critical power nonlinearity," Proc. Am. Math. Soc. 111(2), 487-496 (1991).

15 T. Özsarı, V. Kalantarov, and I. Lasiecka, "Uniform decay rates for the energy of weakly damped defocusing semilinear Schrödinger equations with inhomogeneous Dirichlet boundary control," J. Differ. Equations 251(7), 1841-1863 (2011).

16 T. Özsarı, "Weakly-damped focusing nonlinear Schrödinger equations with Dirichlet control," J. Math. Anal. Appl. 389(1), 84-97 (2012).

17 T. Özsarı, "Global existence and open loop exponential stabilization of weak solutions for nonlinear Schrödinger equations with localized external Neumann manipulation,” Nonlinear Anal. 80, 179-193 (2013).

${ }^{18}$ L. Pitaevskii and S. Stringari, Bose-Einstein Condensation (Clarendon, Oxford, 2003).

${ }^{19}$ W. A. Strauss, "Decay and asymptotics for $\square u=F(u)$," J. Funct. Anal. 2, 409-457 (1968). 
${ }^{20}$ C. Sulem and P. L. Sulem, “The nonlinear Schrödinger equation: Self-focusing and wave collapse,” in Applied Mathematical Sciences (Springer, New York, 1999), Vol. 139.

${ }^{21}$ M. Tsutsumi, "On global solutions to the initial-boundary value problem for the damped nonlinear Schrödinger equations," J. Math. Anal. Appl. 145(2), 328-341 (1990).

${ }^{22}$ M. Tsutsumi, "Nonexistence of global solutions to the Cauchy problem for the damped nonlinear Schrödinger equations," SIAM J. Math. Anal. 15(2), 357-366 (1984).

${ }^{23}$ V. E. Zakharov and A. B. Shabat, "Exact theory of two dimensional self-focusing and one-dimensional self-modulation of waves in nonlinear media," Sov. Phys. JETP 34(1), 62-69 (1972). 